\title{
NONINCREASE OF MUSHY REGION IN A NONHOMOGENEOUS STEFAN PROBLEM
}

\author{
BY \\ I. G. GÖTZ AND B. B. ZALTZMAN
}

Siberian Branch, Academy of Sciences of the USSR, Novosibirsk, USSR

\begin{abstract}
For an arbitrary bounded solution of the Stefan problem the mushy region is nonincreasing in time in a sense of the theory of sets. This result takes place for the nonhomogeneous Stefan problem under some conditions on the behavior of a heat source in the mushy region.
\end{abstract}

Many papers have been devoted during recent years to the study of a mushy region in the Stefan problem. This notion follows from the definition of a generalized solution of the Stefan problem. It characterizes the appearance of the set of nonzero measure, where the temperature of the material coincides with the melting temperature.

The first examples of the appearance of the mushy region in a generalized solution were constructed by Meirmanov [1] and Primicerio [2]. The main cause of it was the presence of the internal heat sources. For a one-dimensional homogeneous Stefan problem, the dynamics of the mushy region were investigated by Meirmanov [3]. $\mathrm{He}$ found the requirements on boundary conditions, producing the disappearance of the mushy region after finite time. The measure of the mushy region in a onephase Stefan problem was estimated by Gustafsson and Mossino [4]. Rogers and Berger [5] made the first attempt to investigate the behavior of the mushy region in a multidimensional two-phase Stefan problem. They proved the nonincrease of the mushy region in the homogeneous Stefan problem with constant Dirichlet data and almost uniformly continuous initial data.

In this paper, we generalize the results of [5]: for an arbitrary bounded solution of the Stefan problem the mushy region is nonincreasing in time in a sense of the theory of sets. This result takes place for the nonhomogeneous Stefan problem under some conditions on the behavior of a heat source in the mushy region.

We deal with a bounded generalized solution of the Stefan problem:

$$
\begin{aligned}
& U_{t}-\Delta \Theta=f(U) \text { in domain } Q_{T}=\Omega \times(0, T), \\
& \left.\Theta\right|_{\partial \Omega \times(0, T)}=\bar{\Theta}(x, t), \\
& \left.U\right|_{t=0}=U_{0}(x) .
\end{aligned}
$$

Received January 23, 1990.

(C)1991 Brown University 
Here $\Omega \subset R^{N}$ is the bounded domain with piecewise smooth boundary $\partial \Omega$, and $\Theta=\chi(U)$, where $\chi$ is the nondecreasing Lipschitz-continuous function with $\chi(U)=0$ when $U \in(-L, 0)$.

By mushy zone in physical variables we shall understand a set

$$
\begin{gathered}
M=\{(x, t): x \in \Omega, t \in(0, T), U(x, t) \in(-L, 0)\}, \\
M\left(t_{0}\right)=M \cap\left\{t=t_{0}\right\} .
\end{gathered}
$$

ThEOREM. Assume that the function $f$ is uniformly Lipschitz-continuous:

$$
\left|f\left(s_{1}\right)-f\left(s_{2}\right)\right| \leq f_{1}\left|s_{1}-s_{2}\right| \text { for every } s_{1}, s_{2} \in R .
$$

Then the following statements are equivalent:

(i) $f(0) \geq 0, f(-L) \leq 0$;

(ii) for every $\bar{\Theta} \in L_{\infty}(\partial \Omega \times(0, T)), U_{0} \in L_{\infty}(\Omega)$ the mushy region in a bounded generalized solution of the problem (1) is nonincreasing in time,

$$
M\left(t_{2}\right) \subset M\left(t_{1}\right) \text { for every } t_{2}>t_{1},
$$

in a sense that $\left|M\left(t_{2}\right) \backslash M\left(t_{1}\right)\right|=0$.

Proof. A logical statement (ii) $\Rightarrow$ (i) is obtained as a corollary of results [1] and [2] about the appearance of the mushy region in the nonhomogeneous Stefan problem.

The proof of the main statement of the theorem is based on the consideration of the time-discrete Stefan problem with smooth initial data:

$$
\begin{aligned}
& \frac{U^{n+1}-U^{n}}{\tau}-\Delta \chi\left(U^{n+1}\right)=f\left(U^{n+1}\right), \quad n=0, \ldots, N-1 ; \tau=T / N, \\
& \left.\Theta^{n}\right|_{\partial \Omega \times(0, T)}=\frac{1}{\tau} \int_{\tau n}^{\tau(n+1)} \bar{\Theta}(x, t) d t, \\
& U^{0}=U_{0}(x),
\end{aligned}
$$

with $U_{0} \in C^{1}(\Omega)$ and $\bar{\Theta} \in L^{\infty}(\partial \Omega \times(0, T))$.

Then we consider an auxiliary family of regularized nonlinear discrete problems $\left(2_{\varepsilon}\right)$ obtained by smoothing the function $\chi$. Here approximate functions $\chi_{\varepsilon}$ are twice-continuously differentiable monotonous functions such that

$$
k \geq \chi_{\varepsilon}^{\prime}(s) \geq \varepsilon, \quad\left|\chi_{\varepsilon}(s)-\chi(s)\right| \leq C \varepsilon, \quad \text { for every } \varepsilon \in R .
$$

The problem $\left(2_{\varepsilon}\right)$ has the classical solution

$$
U_{\varepsilon}(x, t)=U_{\varepsilon}^{n+1}(x) \text { for } t \in[\tau n, \tau(n+1)], n=0,1, \ldots, N-1,
$$

and $U_{\varepsilon}^{n} \in C^{2}\left(\Omega^{\gamma}\right)$ for every $\gamma>0$, where $\Omega^{\gamma}=\{x \in \Omega: \rho(x, \partial \Omega)>\gamma\}$, if $\tau f_{1}<1$. Using the standard methods we obtain estimates uniform in $\varepsilon, \tau$ :

$$
\left\|U_{\varepsilon}\right\|_{L^{\infty}(Q)} \leq C_{1}, \quad\left\|\nabla \Theta_{\varepsilon}\right\|_{L_{2}\left(\Omega^{\prime} \times(0, T)\right)} \leq C_{2}(\gamma)
$$

or every $\gamma>0$. 
Lemma 1. The estimate

$$
\int_{\Omega^{\gamma}}\left|\nabla U_{\varepsilon}^{n}\right| d x \leq C_{3}(\gamma) \text { for every } n \geq 0, \varepsilon>0
$$

is valid.

Proof of Lemma 1. Let us differentiate the regularized equation $\left(2_{\varepsilon}\right)$ with respect to $x_{k}: v=\partial U_{\varepsilon} / \partial x_{k}$,

$$
\left(v^{n+1}-v^{n}\right) / \tau-\Delta\left(\chi_{\varepsilon}^{\prime} v^{n+1}\right)=f^{\prime} v^{n+1} .
$$

Multiplying this by $\varphi \cdot \operatorname{sign}^{\delta} v^{n+1}=\varphi \cdot v^{n+1} /\left(\left(v^{n+1}\right)^{2}+\delta\right)^{1 / 2}$, where $\varphi \geq 0$ and $\varphi \in \dot{C}^{2}\left(\Omega^{\gamma}\right)$, we integrate it with respect to $x$ and sum with respect to $n$ :

$$
\begin{aligned}
\int_{\Omega} \varphi & \sum_{n=0}^{m}\left(v^{n+1}-v^{n}\right) \operatorname{sign}^{\delta} v^{n+1} d x+\tau \sum_{n=0}^{m} \int_{\Omega} \nabla\left(\chi_{\varepsilon}^{\prime} v^{n+1}\right) \nabla\left(\operatorname{sign}^{\delta} v^{n+1} \varphi\right) d x \\
& =\tau \int \sum_{n=0}^{m} f^{\prime} v^{n+1} \operatorname{sign}^{\delta} v^{n+1} \varphi d x .
\end{aligned}
$$

The first term in the left side of this identity, denoted by $I_{1}^{\delta}$, may be rewritten as follows:

$$
I_{1}^{\delta}=\int_{\Omega} \varphi\left(v^{m+1} \operatorname{sign}^{\delta} v^{m+1}-v^{0} \operatorname{sign}^{\delta} v^{1}+\sum_{n=1}^{m} v^{n}\left(\operatorname{sign}^{\delta} v^{n}-\operatorname{sign}^{\delta} v^{n+1}\right)\right) d x .
$$

Taking the limit as $\delta \rightarrow 0$, we easily obtain that

$$
\lim _{\delta \rightarrow 0} I_{1}^{\delta} \geq \int_{\Omega}\left(\left|v^{m+1}\right|-\left|v^{0}\right|\right) \varphi d x .
$$

Applying integration by parts, we can rewrite the second term $\left(I_{2}^{\delta}\right)$ as follows:

$$
\begin{aligned}
I_{2}^{\delta}= & \tau \sum_{n=0}^{m} \int_{\Omega}\left(v^{n+1} \cdot\left(\nabla \operatorname{sign}^{\delta} v^{n+1} \cdot \nabla \chi_{\varepsilon}^{\prime}\right) \cdot \varphi+\chi_{\varepsilon}^{\prime} \cdot \nabla v^{n+1}\right. \\
& \left.\cdot \nabla \operatorname{sign}^{\delta} v^{n+1} \cdot \varphi-\chi_{\varepsilon}^{\prime} \cdot v^{n+1} \cdot \operatorname{div}\left(\operatorname{sign}^{\delta} v^{n+1} \cdot \nabla \varphi\right)\right) d x \\
= & J_{1}^{\delta}+J_{2}^{\delta}+J_{3}^{\delta} .
\end{aligned}
$$

Using the definition of the function $\operatorname{sign}^{\delta}$ we rewrite $J_{1}^{\delta}$ :

$$
J_{1}^{\delta}=\tau \sum_{n=0}^{m} \int_{\Omega} \frac{v^{n+1} \delta}{\left(\left(v^{n+1}\right)^{2}+\delta\right)^{3 / 2}} \cdot \nabla v^{n+1} \cdot \nabla \chi_{\varepsilon}^{\prime} \cdot \varphi d x
$$

The function $F^{\delta}=v^{n+1} \delta /\left(\left(v^{n+1}\right)^{2}+\delta\right)^{3 / 2}$ is bounded uniform in $\delta$,

$$
\left|F^{\delta}\right| \leq \frac{\left|v^{n+1} \delta\right|}{\left(\max \left(\left|v^{n+1}\right|, \sqrt{\delta}\right)\right)^{3}} \leq 1,
$$

and $\lim _{\delta \rightarrow 0} F^{\delta}(x)=0$ for every $x \in \Omega$. Therefore, using that the functions $\nabla v^{n+1}$ and $\nabla \chi_{\varepsilon}^{\prime}$ are bounded in $\Omega^{\gamma}$ we find that $\lim _{\delta \rightarrow 0} J_{1}^{\delta}=0$. 
The term $J_{2}^{\delta}$ is not less than zero, and the term $J_{3}^{\delta}$ may be rewritten as follows:

$$
J_{3}^{\delta}=-\tau \int_{\Omega}\left(\sum_{n=0}^{m} \chi_{\varepsilon}^{\prime} v^{n+1} \nabla \operatorname{sign}^{\delta} v^{n+1} \cdot \nabla \varphi+\chi_{\varepsilon}^{\prime} v^{n+1} \Delta \varphi \operatorname{sign}^{\delta} v^{n+1}\right) d x .
$$

Thus, we have

$$
\lim _{\delta \rightarrow 0} J_{3}^{\delta} \geq-\sum_{n=0}^{m} \tau \int_{\Omega}\left|\nabla \Theta^{n+1}\right||\Delta \varphi| d x \geq-C_{3}(\gamma)
$$

and

$$
\lim _{\delta \rightarrow 0} I_{2}^{\delta} \geq-C_{3}(\gamma)
$$

Finally, we obtain the estimate

$$
\int_{\Omega}\left|v^{m+1}\right| \varphi d x \leq C_{4}+\int_{\Omega} \tau \sum_{n=0}^{m} \varphi f_{1}\left|v^{n+1}\right| d x+\int_{\Omega}\left|v^{0}\right| \varphi d x
$$

and, consequently,

$$
\int_{\Omega}\left|\nabla U_{\varepsilon}^{n}\right| \varphi d x \leq C_{5} \quad \text { for every } n \geq 0, \varepsilon>0,
$$

which yields the estimate (4) and completes the proof of Lemma 1.

With the help of (3), (4) we can take the limit as the regularized parameter $\varepsilon \rightarrow 0$. As a result we obtain the solution $U_{\tau}$ of the time-discrete Stefan problem (2) on which the analogue to (3) holds and

$$
\max _{i=0, N}\left\|U^{i}\right\|_{\mathrm{BV}\left(\Omega^{\gamma}\right)} \leq C_{6}(\gamma) \text { for every } \gamma>0 \text {. }
$$

This estimate follows from (4). It means that the first-order partial derivatives of the functions $U^{i}$ are the bounded measure in $\Omega^{\gamma}$.

Then we shall prove the main statement $[(\mathrm{i}) \Rightarrow$ (ii)] of the theorem for the problem (2). Since the function $\chi\left(U^{n+1}\right)$ satisfies the Poisson equation with a bounded righthand side, then

$$
\chi\left(U^{n+1}\right) \in W_{2, \text { loc }}^{2}(\Omega), \quad n=0, \ldots, N-1 .
$$

Thus, we have

$$
\Delta \chi\left(U^{n+1}\right)=0 \text { and } U^{n+1}=U^{n}+\tau f\left(U^{n+1}\right)
$$

almost everywhere in the set $\left\{x \in \Omega: U^{n+1}(x) \in(-L, 0)\right\}$.

We denote

$$
M^{n+1}=\left\{x \in \Omega: U^{n+1}(x) \in(-L, 0), \Delta \chi\left(U^{n+1}(x)\right)=0\right\} .
$$

Let $x$ be an arbitrary point from $M^{n+1}$. We suppose that $U^{n}(x) \notin(-L, 0)$, for example, $U^{n}(x) \geq 0$. Then

$$
U^{n+1}(x) \geq \tau\left(f\left(U^{n+1}(x)\right)-f(0)\right) \geq-\tau f_{1}\left|U^{n+1}(x)\right|
$$

and $1 \leq \tau f_{1}$. Choosing $\tau<1 / f_{1}$, as above, we obtain the contradiction with the latter expression. Thus, $U^{n}(x) \in(-L, 0)$ and the measure of $M^{n+1} \backslash M^{n}$ is equal 
to zero. This completes the proof of the main statement of the theorem for the time-discrete Stefan problem (2).

Let $u_{\tau}$ be a linear interpolation along time of the function $U_{\tau}$ :

$$
u_{\tau}(x, t)=U^{n+1}(x) \frac{t-n \tau}{\tau}+U^{n}(x) \frac{(n+1) \tau-t}{\tau}, \quad t \in[n \tau,(n+1) \tau] .
$$

The results of [6] and estimates (3), (5) yield

$$
\int_{\Omega^{\gamma}}\left|u_{\tau}(x, t+\Delta t)-u_{\tau}(x, t)\right| d x \leq C_{4}(\gamma) \Delta t^{1 / 2} \text { for every } \gamma>0 .
$$

With the help of (5), (6) we can choose the subsequence $u_{\beta}$ such that

$$
\lim _{\beta \rightarrow 0} u_{\beta}=\lim _{\beta \rightarrow 0} U_{\beta}=U(x, t)
$$

in the norms of the spaces $C^{\alpha}\left(0, T ; L_{1}\left(\Omega^{\gamma}\right)\right), \alpha<1 / 2$, and $L_{2}\left(Q_{T}\right)$. Therefore, taking the limit as $\beta \downarrow 0$ in the integral identity

$$
\int_{Q} \int_{T}\left[u_{\beta} \varphi_{t}+\chi\left(U_{\beta}\right) \Delta \varphi+f\left(U_{\beta}\right) \varphi\right] d x d t=-\int_{\Omega} U_{0} \varphi d x+\int_{0}^{T} \int_{\partial \Omega} \bar{\Theta} \frac{\partial \varphi}{\partial n} d s d t
$$

for any $\varphi$ from $C^{2,1}\left(Q_{T}\right)$ such that $\varphi(x, T)=0$ and $\left.\varphi\right|_{\partial \Omega \times(0, T)}=0$, we observe the function $U$ to be a generalized solution of the Stefan problem (1).

Since the sequence $U_{\tau}$ converges to the function $U$ uniformly over the section $\{t=$ const $\}$ we complete the proof of the main statement of the theorem for the problem (1) with smooth initial data.

We may abandon a requirement as to the smoothness of initial data with the help of the following lemma.

Lemma 2. The generalized solution $U$ is stable in the space $L_{1}(\Omega)$ with respect to the variation of the initial data: If $\widetilde{U}(x, t)$ is the solution of Eq. (1) with the boundary-initial data

$$
\left.\Theta\right|_{\partial \Omega \times(0, T)}=\bar{\Theta},\left.\quad \widetilde{U}\right|_{t=0}=\widetilde{U}_{0}(x) \in L^{\infty}(\Omega),
$$

then

$$
\int_{\Omega}|\tilde{U}(x, t)-U(x, t)| d x \leq e^{f_{1} t} \int_{\Omega}\left|\widetilde{U}_{0}(x)-U_{0}(x)\right| d x .
$$

Corollary 1 . Suppose $\bar{\Theta} \in L^{\infty}(\partial \Omega \times(0, T)), U_{0} \in L_{\infty}(\Omega)$, and the requirements of the theorem and statement (i) for the function $f$ are fulfilled. Then the mushy region of the bounded generalized solution $U$ of the problem (1) may be described in the following way: there exists a nonnegative function $G: \Omega \rightarrow R \cup\{+\infty\}$ such that

$$
M=\{(x, t): x \in M(0), 0 \leq t<G(x)\} .
$$

For every $x \in M(0)$ on the interval $t \in[0, G(x))$ the function $U$ is a solution of the Cauchy problem:

$$
U_{t}=f(U),\left.\quad U\right|_{t=0}=U_{0}(x) .
$$

The first statement of Corollary 1 follows immediately from the theorem. We prove the second statement, at first, as in the theorem, for the time-discrete Stefan 
problem (2). The convergence of the sequence $U_{\tau}$ to the solution $U$ completes the proof of Corollary 1.

Using Corollary 1 we can sometimes find the upper bound of the function $G$, i.e., show that the mushy region has disappeared after a finite time.

Corollary 2. Let the requirements of Corollary 1 be fulfilled and let there exist $s_{0} \in(-L, 0)$ and $\alpha>0$ such that $f(s)>\alpha$ for every $s \in\left(s_{0}, 0\right)$ and $U_{0}(x)>s_{0}$ for every $x \in M(0)$. Then $G(x) \leq-s_{0} / \alpha$.

\section{REFERENCES}

[1] A. M. Meirmanov, An example of nonexistence of a classical solution of the Stefan problem, Dokl. Akad. Nauk SSSR 258, no. 3, 547-549 (1981); English transl., Soviet Math. Dokl. 23, no. 3, 564-566 (1981)

[2] M. Primicerio, Mushy region in phase-change problem, Applied Nonlinear Functional Analysis, Lang, Frankfurt/Main, 1982, pp. 251-269

[3] A. M. Meirmanov, The structure of a generalized solution of the Stefan problem. Periodic solutions, Dokl. Akad. Nauk SSSR 272, no. 4, 789-791 (1983); English transl., Soviet Math. Dokl. 28, no. 2, 440-443 (1983).

[4] B. Gustafsson and J. Mossino, Quelques inégalités isopérimétriques pour le problème de Stefan, C.R. Acad. Sci. Paris Sér. I Math. 305, 669-672 (1987)

[5] J. C. W. Rogers and A. E. Berger, Some properties of the nonlinear semigroup for the problem $u_{t}-\Delta f(u)=0$, Nonlinear Anal., Theory Methods \& Applications 8, 909-939 (1984)

[6] S. N. Kruzhkov, First-order multidimensional quasilinear equations, Mat. Sb. 81, no. 2, 228-255 (1970) 